\title{
An Improved Pseudo-random Generator Based on Discrete Log
}

\author{
Rosario Gennaro \\ IBM T.J.Watson Research Center, P.O. Box 704, Yorktown Heights, NY 10598, \\ rosario@watson.ibm.com
}

\begin{abstract}
Under the assumption that solving the discrete logarithm problem modulo an $n$-bit prime $p$ is hard even when the exponent is a small $c$-bit number, we construct a new and improved pseudo-random bit generator. This new generator outputs $n-c-1$ bits per exponentiation with a $c$-bit exponent.

Using typical parameters, $n=1024$ and $c=160$, this yields roughly 860 pseudo-random bits per small exponentiations. Using an implementation with quite small precomputation tables, this yields a rate of more than 20 bits per modular multiplication, thus much faster than the the squaring (BBS) generator with similar parameters.
\end{abstract}

\section{Introduction}

Many (if not all) cryptographic algorithms rely on the availability of truly random bits. However perfect randomness is a scarce resource. Fortunately for almost all cryptographic applications, it is sufficient to use pseudo-random bits, i.e. sources of randomness that "look" sufficiently random to the adversary.

This notion can be made more formal. The concept of cryptographically strong pseudo-random bit generators (PRBG) was introduced in papers by Blum and Micali [4] and Yao 20]. Informally a PRBG is cryptographically strong if it passes all polynomial-time statistical tests or, in other words, if the distribution of sequences output by the generator cannot be distinguished from truly random sequences by any polynomial-time judge.

Blum and Micali [4] presented the first cryptographically strong PRBG under the assumption that modular exponentiation modulo a prime $p$ is a one-way function. This breakthrough result was followed by a series of papers that culminated in [8] where it was shown that secure PRBGs exists if any one-way function does.

To extract a single pseudo-random bit, the Blum-Micali generator requires a full modular exponentiation in $Z_{p}^{*}$. This was improved by Long and Wigderson [14] and Peralta [17, who showed that up to $O(\log \log p)$ bits could be extracted by a single iteration (i.e. a modular exponentiation) of the Blum-Micali generator. Håstad et al. [10] show that if one considers discrete-log modulo a composite then almost $n / 2$ pseudo-random bits can be extracted per modular exponentiation.

M. Bellare (Ed.): CRYPTO 2000, LNCS 1880, pp. 469481, 2000.

(C) Springer-Verlag Berlin Heidelberg 2000 
Better efficiency can be gained by looking at the quadratic residuosity problem in $Z_{N}^{*}$ where $N$ is a Blum integer (i.e. product of two primes of identical size and both $\equiv 3 \bmod 4$.) Under this assumption, Blum et al. [3] construct a secure PRBG for which each iteration consists of a single squaring in $Z_{N}^{*}$ and outputs a pseudo-random bit. Alexi et al. 2] showed that one can improve this to $O(\log \log N)$ bits and rely only the intractability of factoring as the underlying assumption. Up to this date, this is the most efficient provably secure PRBG.

In 16. Patel and Sundaram propose a very interesting variation on the BlumMicali generator. They showed that if solving the discrete log problem modulo an $n$-bit prime $p$ is hard even when the exponent is small (say only $c$ bits long with $c<n$ ) then it is possible to extract up to $n-c-1$ bits from one iteration of the Blum-Micali generator. However the iterated function of the generator itself remains the same, which means that one gets $n-c-1$ bits per full modular exponentiations. Patel and Sundaram left open the question if it was possible to modify their generator so that each iteration consisted of an exponentiation with a small $c$-bit exponent. We answer their question in the affirmative.

Our Contribution. In this paper we show that it is possible to construct a high-rate discrete-log based secure PRBG. Under the same assumption introduced in [16] we present a generator that outputs $n-c-1$ bits per iteration, which consists of a single exponentiation with a $c$-bit exponent.

The basic idea of the new scheme is to show that if the function $f:\{0,1\}^{c} \longrightarrow$ $Z_{p}^{*}$ defined as $f(x)=g^{x} \bmod p$ is is a one-way function then it also has also strong pseudo-randomness properties over $Z_{p}^{*}$. In particular it is possible to think of it as pseudo-random generator itself. By iterating the above function and outputting the appropriate bits, we obtain an efficient pseudo-random bit generator.

Another attractive feature of this generator (which is shared by the BlumMicali and Patel-Sundaram generators as well) is that all the exponentiations are computed over a fixed basis, and thus precomputation tables can be used to speed them up.

Using typical parameters $n=1024$ and $c=160$ we obtain roughly 860 pseudo-random bits per 160-bit exponent exponentiations. Using the precomputation scheme proposed in 13 one can show that such exponentiation will cost on average roughly 40 multiplications, using a table of only 12 Kbytes. Thus we obtain a rate of more than 21 pseudo-random bits per modular multiplication. Different tradeoffs between memory and efficiency can be obtained.

\section{Preliminaries}

In this section we summarize notations, definitions and prior work which is relevant to our result. In the following we denote with $\{0,1\}^{n}$ the set of $n$-bit strings. If $x \in\{0,1\}^{n}$ then we write $x=x_{n} x_{n-1} \ldots x_{1}$ where each $x_{i} \in\{0,1\}$. If we think of $x$ as an integer then we have $x=\sum_{i} x_{i} 2^{i-1}$ (that is $x_{n}$ is the most significant bit). With $R_{n}$ we denote the uniform distribution over $\{0,1\}^{n}$. 


\subsection{Pseudo-random Number Generators}

Let $X_{n}, Y_{n}$ be two arbitrary probability ensembles over $\{0,1\}^{n}$. In the following we denote with $x \leftarrow X_{n}$ the selection of an element $x$ in $\{0,1\}^{n}$ according to the distribution $X_{n}$.

We say that $X_{n}$ and $Y_{n}$ have statistical distance bounded by $\Delta(n)$ if the following holds:

$$
\sum_{x \in\{0,1\}^{n}}\left|\operatorname{Prob}_{X_{n}}[x]-\operatorname{Prob}_{Y_{n}}[x]\right| \leq \Delta(n)
$$

We say that $X_{n}$ and $Y_{n}$ are statistically indistinguishable if for every polynomial $P(\cdot)$ and for sufficiently large $n$ we have that

$$
\Delta(n) \leq \frac{1}{P(n)}
$$

We say that $X_{n}$ and $Y_{n}$ are computationally indistinguishable (a concept introduced in [7]) if any polynomial time machine cannot distinguish between samples drawn according to $X_{n}$ or according to $Y_{n}$. More formally:

Definition 1. Let $X_{n}, Y_{n}$ be two families of probability distributions over $\{0,1\}^{n}$. Given a Turing machine $\mathcal{D}$ consider the following quantities

$$
\begin{gathered}
\delta_{\mathcal{D}, X_{n}}=\operatorname{Prob}\left[x \leftarrow X_{n} ; \mathcal{D}(x)=1\right] \\
\delta_{\mathcal{D}, Y_{n}}=\operatorname{Prob}\left[y \leftarrow Y_{n} ; \mathcal{D}(y)=1\right]
\end{gathered}
$$

We say that $X_{n}$ and $Y_{n}$ are computationally indistinguishable if for every probabilistic polynomial time $\mathcal{D}$, for every polynomial $P(\cdot)$, and for sufficiently large $n$ we have that

$$
\left|\delta_{\mathcal{D}, X_{n}}-\delta_{\mathcal{D}, Y_{n}}\right| \leq \frac{1}{P(n)}
$$

We now move to define pseudo-random number generators [420]. There are several equivalent definitions, but the following one is sufficient for our purposes. Consider a family of functions

$$
\mathrm{G}_{n}:\{0,1\}^{k_{n}} \longrightarrow\{0,1\}^{n}
$$

where $k_{n}<n . \mathrm{G}_{n}$ induces a family of probability distributions (which we denote with $G_{n}$ ) over $\{0,1\}^{n}$ as follows

$$
\operatorname{Prob}_{G_{n}}[y]=\operatorname{Prob}\left[y=\mathrm{G}_{n}(s) ; s \leftarrow R_{k_{n}}\right]
$$

Definition 2. We say that $\mathrm{G}_{n}$ is a cryptographically strong pseudo-random bit generator if the function $\mathrm{G}_{n}$ can be computed in polynomial time and the two families of probability distributions $R_{n}$ and $G_{n}$ are computationally indistinguishable.

The input of a pseudo-random generator is usually called the seed. 


\subsection{Pseudo-randomness over Arbitrary Sets}

Let $A_{n}$ be a family of sets such that for each $n$ we have $2^{n-1} \leq\left|A_{n}\right|<2^{n}$ (i.e. we need $n$ bits to describe elements of $A_{n}$ ). We denote with $U_{n}$ the uniform distribution over $A_{n}$. Also let $k_{n}$ be a sequence of numbers such that for each $n, k_{n}<n$. Consider a family of functions

$$
\mathrm{AG}_{n}:\{0,1\}^{k_{n}} \longrightarrow A_{n}
$$

$\mathrm{AG}_{n}$ induces a family of probability distributions (which we denote with $A G_{n}$ ) over $A_{n}$ as follows

$$
\operatorname{Prob}_{A G_{n}}[y]=\operatorname{Prob}\left[y=\mathrm{AG}_{n}(s) ; s \leftarrow R_{k_{n}}\right]
$$

Definition 3. We say that $\mathrm{AG}_{n}$ is a cryptographically strong pseudo-random generator over $A_{n}$ if the function $\mathrm{AG}_{n}$ can be computed in polynomial time and the two families of probability distributions $U_{n}$ and $A G_{n}$ are computationally indistinguishable.

A secure pseudo-random generator over $A_{n}$ is already useful for applications in which one needs pseudo-random elements of that domain. Indeed no adversary will be able to distinguish if $y \in A_{n}$ was truly sampled at random or if it was computed as $\mathrm{AG}_{n}(s)$ starting from a much shorter seed $s$. An example of this is to consider $A_{n}$ to be $Z_{p}^{*}$ for an $n$-bit prime number $p$. If our application requires pseudo-random elements of $Z_{p}^{*}$ then such a generator would be sufficient.

However as bit generators they may not be perfect, since if we look at the bits of an encoding of the elements of $A_{n}$, then their distribution may be biased. This however is not going to be a problem for us since we will use pseudo-random generators over arbitrary sets as a tool in the proof of our main pseudo-random bit generator.

\subsection{The Discrete Logarithm Problem}

Let $p$ be a prime. We denote with $n$ the binary length of $p$. It is well known that $Z_{p}^{*}=\{x: 1 \leq x \leq p-1\}$ is a cyclic group under multiplication $\bmod p$. Let $g$ be a generator of $Z_{p}^{*}$. Thus the function

$$
\begin{aligned}
& f: Z_{p-1} \longrightarrow Z_{p}^{*} \\
& f(x)=g^{x} \bmod p
\end{aligned}
$$

is a permutation. The inverse of $f$ (called the discrete logarithm function) is conjectured to be a function hard to compute (the cryptographic relevance of this conjecture first appears in the seminal paper by Diffie and Hellman [5] on public-key cryptography). The best known algorithm to compute discrete logarithms is the so-called index calculus method [1] which however runs in time sub-exponential in $n$. 
In some applications (like the one we are going to describe in this paper) it is important to speed up the computation of the function $f(x)=g^{x}$. One possible way to do this is to restrict its input to small values of $x$. Let $c$ be a integer which we can think as depending on $n(c=c(n))$. Assume now that we are given $y=g^{x} \bmod p$ with $x \leq 2^{c}$. It appears to be reasonable to assume that computing the discrete logarithm of $y$ is still hard even if we know that $x \leq 2^{c}$. Indeed the running time of the index-calculus method depends only on the size $n$ of the whole group. Depending on the size of $c$, different methods may actually be more efficient. Indeed the so-called baby-step giant-step algorithm by Shanks [12] or the rho algorithm by Pollard [18] can compute the discrete log of $y$ in $O\left(2^{c / 2}\right)$ time.

Thus if we set $c=\omega(\log n)$, there are no known polynomial time algorithms that can compute the discrete $\log$ of $y=g^{x} \bmod p$ when $x \leq 2^{c}$. In [16] it is explicitly assumed that no such efficient algorithm can exist. This is called the Discrete Logarithm with Short c-Bit Exponents (c-DLSE) Assumption and we will adopt it as the basis of our results as well.

Assumption 1 (c-DLSE [16]) Let PRIMES(n) be the set of n-bit primes and let $c$ be a quantity that grows faster than $\log n$ (i.e. $c=\omega(\log n)$ ). For every probabilistic polynomial time Turing machine $\mathcal{I}$, for every polynomial $P(\cdot)$ and for sufficiently large $n$ we have that

$$
\operatorname{Pr}\left[\begin{array}{l}
p \leftarrow P R I M E S(n) ; \\
x \leftarrow R_{c} ; \\
\mathcal{I}\left(p, g, g^{x}, c\right)=x
\end{array}\right] \leq \frac{1}{P(n)}
$$

This assumption is somewhat supported by a result by Schnorr 19] who proves that no generic algorithm can compute $c$-bits discrete logarithms in less than $2^{c / 2}$ generic steps. A generic algorithm is restricted to only perform group operations and cannot take advantage of specific properties of the encoding of group elements.

In practice, given today's computing power and discrete-log computing algorithms, it seems to be sufficient to set $n=1024$ and $c=160$. This implies a "security level" of $2^{80}$ (intended as work needed in order to "break" 160-DLSE).

\subsection{Hard Bits for Discrete Logarithm}

The function $f(x)=g^{x} \bmod p$ is widely considered to be one-way (i.e. a function easy to compute but not to invert). It is well known that even if $f$ is a one-way function, it does not hide all information about its preimages. For the specific case of the discrete logarithm, it is well known that given $y=g^{x} \bmod p$ it is easy to guess the least significant bit of $x \in Z_{p-1}$ by testing to see if $y$ is a quadratic residue or not in $Z_{p}^{*}$ (there is a polynomial-time test to determine that).

A Boolean predicate $\Pi$ is said to be hard for a one-way function $f$ if any algorithm $\mathcal{A}$ that given $y=f(x)$ guesses $\Pi(x)$ with probability substantially better than $1 / 2$, can be used to build another algorithm $\mathcal{A}^{\prime}$ that on input $y$ computes $x$ with non-negligible probability. 
Blum and Micali in [4] prove that the predicate

$$
\begin{aligned}
& \Pi: Z_{p-1} \longrightarrow\{0,1\} \\
& \Pi(x)=\left(x \leq \frac{p-1}{2}\right)
\end{aligned}
$$

is hard for the discrete logarithm function. Recently Håstad and Näslund [9] proved that every bit of the binary representation of $x$ (except the least significant one) is hard for the discrete log function.

In terms of simultaneous security of several bits, Long and Wigderson 14 and Peralta 17] showed that there are $O(\log \log p)$ predicates which are simultaneously hard for discrete log. Simultaneously hard means that the whole collection of bits looks "random" even when given $y=g^{x}$. A way to formalize this (following [20]) is to say that it is not possible to guess the value of the $j^{\text {th }}$ predicate even after seeing $g^{x}$ and the value of the previous $j-1$ predicates over $x$. Formally: there exists $O(\log \log p)$ Boolean predicates

$$
\Pi_{i}: Z_{p-1} \longrightarrow\{0,1\} \quad \text { for } i=1, \ldots, O(\log \log p)
$$

such that for every $1 \leq j \leq O(\log \log p)$, if there exists a probabilistic polynomialtime algorithm $\mathcal{A}$ and a polynomial $P(\cdot)$ such that

$$
\operatorname{Prob}\left[x \leftarrow Z_{p-1} ; \mathcal{A}\left(g^{x}, \Pi_{1}(x), \ldots, \Pi_{j-1}(x)\right)=\Pi_{j}(x)\right] \geq \frac{1}{2}+\frac{1}{P(n)}
$$

then there exists a probabilistic polynomial time algorithm $\mathcal{A}^{\prime}$ which on input $g^{x}$ computes $x$ with non-negligible probability.

\subsection{The Patel-Sundaram Generator}

Let $p$ be a $n$-bit prime such that $p \equiv 3 \bmod 4$ and $g$ a generator of $Z_{p}^{*}$. Denote with $c$ a quantity that grows faster than $\log n$, i.e. $c=\omega(\log n)$.

In [16] Patel and Sundaram prove that under the $c$-DLSE Assumption the bits $x_{2}, x_{3}, \ldots, x_{n-c}$ are simultaneously hard for the function $f(x)=g^{x} \bmod p$. More formally1:

Theorem 1 ([16]). For sufficiently large $n$, if $p$ is a $n$-bit prime such that $p \equiv 3 \bmod 4$ and if the $c$-DLSE Assumption holds, then for every $j, 2 \leq j \leq n-c$, for every polynomial time Turing machine $\mathcal{A}$, for every polynomial $P(\cdot)$ and for sufficiently large $n$ we have that

$$
\left|\operatorname{Prob}\left[x \leftarrow Z_{p-1} ; \mathcal{A}\left(g^{x}, x_{2}, \ldots, x_{j-1}\right)=x_{j}\right]-\frac{1}{2}\right| \leq \frac{1}{P(n)}
$$

$\overline{1}$ We point out that [16] requires that $p$ be a safe prime, i.e. such that $(p-1) / 2$ is also a prime; but a close look at their proof reveals that $p \equiv 3 \bmod 4$ suffices. 
We refer the reader to [16] for a proof of this Theorem.

Theorem 11 immediately yields a secure PRBG. Start with $x^{(0)} \in_{R} Z_{p-1}$. Set $x^{(i)}=g^{x^{(i-1)}} \bmod p$. Set also $r^{(i)}=x_{2}^{(i)}, x_{3}^{(i)}, \ldots, x_{n-c}^{(i)}$. The output of the generator will be $r^{(0)}, r^{(1)}, \ldots, r^{(k)}$ where $k$ is the number of iterations.

Notice that this generator outputs $n-c-1$ pseudo-random bits at the cost of a modular exponentiation with a random $n$-bit exponent.

\section{Our New Generator}

We now show that under the DLSE Assumption it is possible to construct a PRBG which is much faster than the Patel-Sundaram one. In order to do this we first revisit the construction of Patel and Sundaram to show how one can obtain a pseudo-random generator over $Z_{p}^{*} \times\{0,1\}^{n-c-1}$.

Then we construct a function from $Z_{p-1}$ to $Z_{p}^{*}$ which induces a pseudorandom distribution over $Z_{p}^{*}$. The proof of this fact is by reduction to the security of the modified Patel-Sundaram generator. This function is not a generator yet, since it does not stretch its input.

We finally show how to obtain a pseudo-random bit generator, by iterating the above function and outputting the appropriate bits.

\subsection{The Patel-Sundaram Generator Revisited}

As usual let $p$ be a $n$-bit prime, $p \equiv 3 \bmod 4$, and $c=\omega(\log n)$. Consider the following function (which we call PSG for Patel-Sundaramam Generator):

$$
\begin{gathered}
\mathrm{PSG}_{n, c}: Z_{p-1} \longrightarrow Z_{p}^{*} \times\{0,1\}^{n-c-1} \\
\mathrm{PSG}_{n, c}(x)=\left(g^{x} \bmod p, x_{2}, \ldots, x_{n-c}\right)
\end{gathered}
$$

That is, on input a random seed $x \in Z_{p-1}$, the generator outputs $g^{x}$ and $n-c-1$ consecutive bits of $x$, starting from the second least significant.

An immediate consequence of the result in [16, is that under the $c$-DLSE assumption $\mathrm{PSG}_{n, c}$ is a secure pseudo-random generator over the set $Z_{p}^{*} \times$ $\{0,1\}^{n-c-1}$. More formally, if $U_{n}$ is the uniform distribution over $Z_{p}^{*}$, then the distribution induced by $\mathrm{PSG}_{n, c}$ over $Z_{p}^{*} \times\{0,1\}^{n-c-1}$ is computationally indistinguishable from the distribution $U_{n} \times R_{n-c-1}$.

In other words, for any probabilistic polynomial time Turing machine $\mathcal{D}$, we can define

$$
\begin{gathered}
\delta_{\mathcal{D}, U R_{n}}=\operatorname{Prob}\left[y \leftarrow Z_{p}^{*} ; r \leftarrow R_{n-c-1} ; \mathcal{D}(y, r)=1\right] \\
\delta_{\mathcal{D}, P S G_{n, c}}=\operatorname{Prob}\left[x \leftarrow Z_{p-1} ; \mathcal{D}\left(\mathrm{PSG}_{n, c}(x)\right)=1\right]
\end{gathered}
$$

then for any polynomial $P(\cdot)$ and for sufficiently large $n$, we have that

$$
\left|\delta_{\mathcal{D}, U R_{n}}-\delta_{\mathcal{D}, P S G_{n, c}}\right| \leq \frac{1}{P(n)}
$$

In the next section we show our new generator and we prove that if it is not secure that we can show the existence of a distinguisher $\mathcal{D}$ that contradicts the above. 


\subsection{A Preliminary Lemma}

We also assume that $p$ is a $n$-bit prime, $p \equiv 3 \bmod 4$ and $c=\omega(\log n)$. Let $g$ be a generator of $Z_{p}^{*}$ and denote with $\hat{g}=g^{2^{n-c}} \bmod p$. Recall that if $s$ is an integer we denote with $s_{i}$ the $i^{t h}$-bit in its binary representation.

The function we consider is the following.

$$
\begin{gathered}
\mathrm{RG}_{n, c}: Z_{p-1} \longrightarrow Z_{p}^{*} \\
\mathrm{RG}_{n, c}(s)=\hat{g}^{\left(s \operatorname{div} 2^{n-c}\right)} g^{s_{1}} \bmod p
\end{gathered}
$$

That is we consider modular exponentiation in $Z_{p}^{*}$ with base $g$, but only after zeroing the bits in positions $2, \ldots, n-c$ of the input $s$ (these bits are basically ignored).

The function RG induces a distribution over $Z_{p}^{*}$ in the usual way. We denote it with $R G_{n, c}$ the following probability distribution over $Z_{p}^{*}$

$$
\operatorname{Prob}_{R G_{n, c}}[y]=\operatorname{Prob}\left[y=\mathrm{RG}_{n, c}(s) ; s \leftarrow Z_{p-1}\right]
$$

The following Lemma states that the distribution $R G_{n, c}$ is computationally indistinguishable from the uniform distribution over $Z_{p}^{*}$ if the $c$-DLSE assumption holds.

Lemma 1. Let $p$ be a $n$-bit prime, with $p \equiv 3 \bmod 4$ and let $U_{n}$ be the uniform distribution over $Z_{p}^{*}$. If the c-DLSE Assumption holds, then the two distributions $U_{n}$ and $R G_{n, c}$ are computationally indistinguishable (see Definition 11).

The proof of the Lemma goes by contradiction. We show that if $R G_{n, c}$ can be distinguished from $U_{n}$, then the modified Patel-Sundaram generator PSG is not secure. We do this by showing that any efficient distinguisher between $R G_{n, c}$ and the uniform distribution over $Z_{p}^{*}$ can be transformed into a distinguisher for $\mathrm{PSG}_{n, c}$. This will contradict Theorem 1 and ultimately the $c$-DLSE Assumption.

Sketch of Proof Assume for the sake of contradiction that there exists a distinguisher $\mathcal{D}$ and a polynomial $P(\cdot)$ such that for infinitely many $n$ 's we have that

$$
\delta_{\mathcal{D}, U_{n}}-\delta_{\mathcal{D}, R G_{n, c}} \geq \frac{1}{P(n)}
$$

where

$$
\begin{gathered}
\delta_{\mathcal{D}, U_{n}}=\operatorname{Prob}\left[x \leftarrow Z_{p}^{*} ; \mathcal{D}(p, g, x, c)=1\right] \\
\delta_{\mathcal{D}, R G_{n, c}}=\operatorname{Prob}\left[s \leftarrow Z_{p-1} ; \mathcal{D}\left(p, g, \mathrm{RG}_{n, c}(s), c\right)=1\right]
\end{gathered}
$$

We show how to construct a distinguisher $\hat{\mathcal{D}}$ that "breaks" PSG.

In order to break $\mathrm{PSG}_{n, c}$ we are given as input $(p, g, y, r, c)$ with $y \in Z_{p}^{*}$ and $r \in\{0,1\}^{n-c-1}$ and we want to guess if it comes from the distribution $U_{n} \times R_{n-c-1}$ or from the distribution $P S G_{n, c}$ of outputs of the generator $\mathrm{PSG}_{n, c}$. The distinguisher $\hat{\mathcal{D}}$ will follow this algorithm: 
1. Consider the integer $z:=r \circ 0$ where $\circ$ means concatenation. Set $w:=$ $y g^{-z} \bmod p$

2. Output $\mathcal{D}(p, g, w, c)$

Why does this work? Assume that $(y, r)$ was drawn according to $\mathrm{PSG}_{n, c}(x)$ for some random $x \in Z_{p-1}$. Then $w=g^{u}$ where $u=2^{n-c}\left(x \operatorname{div} 2^{n-c}\right)+x_{1} \bmod p-1$. That is, the discrete log of $w$ in base $g$ has the $n-c-1$ bits in position $2, \ldots, n-c$ equal to 0 (this is because $r$ is identical to those $n-c-1$ bits of the discrete log of $y$ by the assumption that $(y, r)$ follows the $P S G_{n, c}$ distribution). Thus once we set $\hat{g}=g^{2^{n-c}}$ we get $w=\hat{g}^{x} \operatorname{div} 2^{n-c} g^{x_{1}} \bmod p$, i.e. $w=\mathrm{RG}_{n, c}(x)$. Thus if $(y, r)$ is drawn according to $P S G_{n}$ then $w$ follows the same distribution as $R G_{n}$.

On the other hand if $(y, r)$ was drawn with $y$ randomly chosen in $Z_{p}^{*}$ and $r$ randomly chosen in $\{0,1\}^{n-c-1}$, then all we know is that $w$ is a random element of $Z_{p}^{*}$.

Thus $\hat{\mathcal{D}}$ will guess the correct distribution with the same advantage as $\mathcal{D}$ does. Which contradicts the security of the PSG generator.

\subsection{The New Generator}

It is now straightforward to construct the new generator. The algorithm receives as a seed a random element $s$ in $Z_{p-1}$ and then it iterates the function RG on it. The pseudo-random bits outputted by the generator are the bits ignored by the function RG. The output of the function RG will serve as the new input for the next iteration.

More in detail, the algorithm $\mathrm{IRG}_{n, c}$ (for Iterated-RG generator) works as follows. Start with $x^{(0)} \in_{R} Z_{p-1}$. Set $x^{(i)}=\mathrm{RG}_{n, c}\left(x^{(i-1)}\right)$. Set also $r^{(i)}=$ $x_{2}^{(i)}, x_{3}^{(i)}, \ldots, x_{n-c}^{(i)}$. The output of the generator will be $r^{(0)}, r^{(1)}, \ldots, r^{(k)}$ where $k$ is the number of iterations (chosen such that $k=\operatorname{poly}(n)$ and $k(n-c-1)>n$ ).

Notice that this generator outputs $n-c-1$ pseudo-random bits at the cost of a modular exponentiation with a random $c$-bit exponent (i.e. the cost of the computation of the function RG).

Theorem 2. Under the c-DLSE Assumption, $\mathrm{IRG}_{n, c}$ is a secure pseudo-random bit generator (see Definition 2 ).

Sketch of Proof We first notice that, for sufficiently large $n, r^{(0)}$ is an almost uniformly distributed $(n-c-1)$-bit string. This is because $r^{(0)}$ is composed of the bits in position $2,3, \ldots, n-c$ of a random element of $Z_{p-1}$ and thus their bias is bounded by $2^{-c}$ (i.e. the statistical distance between the distribution of $r^{(0)}$ and the uniform distribution over $\{0,1\}^{n-c-1}$ is bounded by $2^{-c}$.

Now by virtue of Lemma 1 we know that all the values $x^{(i)}$ follow a distribution which is computationally indistinguishable from the uniform one on $Z_{p}^{*}$. By the same argument as above it follows that all the $r^{(i)}$ must follow a distribution which is computationally indistinguishable from $R_{n-c-1}$. 
More formally, the proof follows a hybrid argument. If there is a distinguisher $\mathcal{D}$ between the distribution induced by $\mathrm{IRG}_{n, c}$ and the distribution $R_{k(n-c-1)}$, then for a specific index $i$ we must have a distinguisher $\mathcal{D}_{1}$ between the distribution followed by $r^{(i)}$ and the uniform distribution $R_{n-c-1}$. Now that implies that it is possible to distinguish the distribution followed by $x^{(i)}$ and the uniform distribution over $Z_{p}^{*}$ (just take the bits in position $2,3, \ldots, n-c$ of the input and pass them to $\mathcal{D}_{2}$ ). This contradicts Lemma 1 and ultimately the $c$-DLSE Assumption.

\section{Efficiency Analysis}

Our new generator is very efficient. It outputs $n-c-1$ pseudo-random bits at the cost of a modular exponentiation with a random $c$-bit exponent, or roughly $1.5 c$ modular multiplications in $Z_{p}^{*}$. Compare this with the Patel-Sundaram generator where the same number of pseudo-random bits would cost $1.5 n$ modular multiplications. Moreover the security of our scheme is tightly related to the security of the Patel-Sundaram one, since the reduction from our scheme to theirs is quite immediate.

So far we have discussed security in asymptotic terms. If we want to instantiate practical parameters we need to analyze more closely the concrete security of the proposed scheme.

A close look at the proof of security in [16] shows the following. If we assume that Theorem 1 fails, i.e. that for some $j, 2 \leq j \leq n-c$, there exists an algorithm $\mathcal{A}$ which runs in time $T(n)$, and a polynomial $P(\cdot)$ such that w.l.o.g.

$$
\operatorname{Prob}\left[x \leftarrow Z_{p-1} ; \mathcal{A}\left(g^{x}, x_{2}, \ldots, x_{j-1}\right)=x_{j}\right]>\frac{1}{2}+\frac{1}{P(n)}
$$

then we have an algorithm $\mathcal{I}^{\mathcal{A}}$ to break $c$-DLSE which runs in time $O((n-$ c) $\left.c P^{2}(n) T(n)\right)$ if $2 \leq j<n-c-\log P(n)$ and in time $O\left((n-c) c P^{3}(n) T(n)\right)$ if $n-c-\log P(n) \leq j \leq n-c$ (the hidden constant is very small). This is a very crude analysis of the efficiency of the reduction in [16] and it is quite possible to improve on it (details in the final paper).

In order to be able to say that the PRBG is secure we need to make sure that the complexity of this reduction is smaller than the time to break $c$-DLSE with the best known algorithm (which we know today is $2^{c / 2}$ ).

COMPARISON WITH THE BBS GENERATOR. The BBS generator was introduced by Blum et al. in [3] under the assumption that deciding quadratic residuosity modulo a composite is hard. The generator works by repeatedly squaring $\bmod N$ a random seed in $Z_{N}^{*}$ where $N$ is a Blum integer $(N=P Q$ with $P, Q$ both primes of identical size and $\equiv 3 \bmod 4$.) At each iteration it outputs the least significant bit of the current value. The rate of this generator is thus of $1 \mathrm{bit} / \mathrm{squaring}$. In [2], Alexi et al. showed that one can output up to $k=O(\log \log N)$ bits per iteration of the squaring generator (and this while also relaxing the underlying 
assumption to the hardness of factoring). The actual number $k$ of bits that can be outputted depends on the concrete parameters adopted.

The 2 reduction is not very tight and it was recently improved by Fischlin and Schnorr in [6]. The complexity of the reduction quoted there is

$$
O\left(n \log n P^{2}(n) T(n)+n^{2} P^{4}(n) \log n\right)
$$

(here $P(n), T(n)$ refers to a machine which guesses the next bit in one iteration of the BBS generator in time $T(n)$ and with advantage $1 / P(n))$.

If we want to output $k$ bits per iteration, the complexity grows by a factor of $2^{2 k}$ and the reduction quickly becomes more expensive than known factoring algorithms. Notice instead that the reduction in [16] (and thus in ours) depends only linearly on the number of bits outputted.

Concrete Parameters. Let's fix $n=1024$ and $c=160$. With these parameters we can safely assume that the complexity of the best known algorithms to break $c$-DLSE [1217] is beyond the reach of today's computing capabilities.

For moduli of size $n=1024$, the results in [6] seem to indicate that in practice, for $n=1024$ we can output around 4 bits per iteration of the BBS generator, if we want to rule out adversaries which run in time $T=1$ MIPS-year (roughly $10^{13}$ instructions) and predict the next bit with advantage $1 / 100$ (which is quite high). This yields a rate of 4 bits per modular squaring.

Using the same indicative parameters suggested in [6], we can see that we can safely output all $n-c-1 \approx 860$ bits in one iteration of the Patel-Sundaram generator. Since the security of our scheme is basically the same as their generator we can also output all 860 bits in our scheme as well.

Thus we obtain 860 bits at the cost of roughly 240 multiplications, which yields a rate of about 3.5 bits per modular multiplication. Thus the basic implementation of our scheme has efficiency comparable to the BBS generator. In the next section we show how to improve on this, by using precomputation tables.

\subsection{Using Precomputed Tables}

The most expensive part of the computation of our generator is to compute $\hat{g}^{s} \bmod p$ where $s$ is a $c$-bit value.

We can take advantage of the fact that in our generator 2 the modular exponentiations are all computed over the same basis $\hat{g}$. This feature allows us to precompute powers of $\hat{g}$ and store them in a table, and then use this values to compute fastly $\hat{g}^{s}$ for any $s$.

The simplest approach is to precompute a table $T$

$$
T=\left\{\hat{g}^{2^{i}} \bmod p ; i=0, \ldots, c\right\}
$$

Now, one exponentiation with base $\hat{g}$ and a random $c$-bit exponent can be computed using only $.5 c$ multiplications on average. The cost is an increase to $O(c n)$

\footnotetext{
${ }^{2}$ As well as in the Patel-Sundaram one, or in the Blum-Micali one
} 
bits of required memory. With this simple improvement one iteration of our generator will require roughly 80 multiplications, which yields a rate of more that 10 pseudo-random bits per multiplication. The size of the table is about 20 Kbytes.

Lim and Lee [13] present more flexible trade-offs between memory and computation time to compute exponentiations over a fixed basis. Their approach is applicable to our scheme as well. In short, the [13] precomputation scheme is governed by two parameters $h, v$. The storage requirement is $\left(2^{h}-1\right) v$ elements of the field. The number of multiplications required to exponentiate to a $c$-bit exponent is $\left\lceil\frac{c}{h}\right\rceil+\left\lceil\frac{c}{h v}\right\rceil-2$ in the worst case.

Using the choice of parameters for 160-bit exponents suggested in 13 we can get roughly 40 multiplications with a table of only 12 Kbytes. This yields a rate of more than 21 pseudo-random bits per multiplication. A large memory implementation (300 Kbytes) will yield a rate of roughly 43 pseudo-random bits per multiplication.

In the final version of this paper we will present a more complete concrete analysis of our new scheme.

\section{Conclusions}

In this paper we presented a secure pseudo-random bit generator whose efficiency is comparable to the squaring (BBS) generator. The security of our scheme is based on the assumption that solving discrete logarithms remains hard even when the exponent is small. This assumption was first used by Patel and Sundaram in [16]. Our construction however is much faster than theirs since it only uses exponentiations with small inputs.

An alternative way to look at our construction is the following. Under the $c$-DLSE assumption the function $f:\{0,1\}^{c} \longrightarrow Z_{p}^{*}$ defined as $f(x)=g^{x}$ is a one-way function. Our results indicate that $f$ has also strong pseudo-randomness properties over $Z_{p}^{*}$. In particular it is possible to think of it as pseudo-random generator itself. We are aware of only one other example in the literature of a one-way function with this properties, in [11] based on the hardness of subsetsum problems.

The DLSE Assumption is not as widely studied as the regular discrete log assumption so it needs to be handled with care. However it seems a reasonable assumption to make.

It would be nice to see if there are other cryptographic primitives that could benefit in efficiency from the adoption of stronger (but not unreasonable) number-theoretic assumptions. Examples of this are already present in the literature (e.g. the efficient construction of pseudo-random functions based on the Decisional Diffie-Hellman problem in [15.) It would be particularly interesting to see a pseudo-random bit generator that beats the rate of the squaring generator, even if at the cost of a stronger assumption on factoring or RSA. 


\section{Acknowledgments}

This paper owes much to the suggestions and advices of Shai Halevi. Thanks also to the other members of the CRYPTO committee for their suggestions and to Dario Catalano for reading early drafts of the paper.

\section{References}

1. L. Adleman. A Subexponential Algorithm for the Discrete Logarithm Problem with Applications to Cryptography. IEEE FOCS, pp.55-60, 1979.

2. W. Alexi, B. Chor, O. Goldreich and C. Schnorr. RSA and Rabin Functions: Certain Parts are as Hard as the Whole. SIAM J. Computing, 17(2):194-209, April 1988.

3. L. Blum, M. Blum and M. Shub. A Simple Unpredictable Pseudo-Random Number Generator. SIAM J.Computing, 15(2):364-383, May 1986.

4. M. Blum and S. Micali. How to Generate Cryptographically Strong Sequences of Pseudo-Random Bits. SIAM J.Computing, 13(4):850-864, November 1984.

5. W. Diffie and M. Hellman. New Directions in Cryptography. IEEE Trans. Inf. Theory, IT-22:644-654, November 1976.

6. R. Fischlin and C. Schnorr. Stronger Security Proofs for RSA and Rabin Bits. J.Crypt., 13(2):221-244, Spring 2000.

7. S. Goldwasser and S. Micali. Probabilistic Encryption. JCSS, 28:270-299, 1988.

8. J. Håstad, R. Impagliazzo, L. Levin and M. Luby. A Pseudo-Random Generator from any One-Way Function. SIAM J.Computing, 28(4):1364-1396, 1999.

9. J. Håstad and M. Näslund. The Security of Individual RSA Bits. IEEE FOCS, pp.510-519, 1998.

10. J. Håstad, A. Schrift and A. Shamir. The Discrete Logarithm Modulo a Composite Hides $O(n)$ Bits. JCSS, 47:376-404, 1993.

11. R. Impagliazzo and M. Naor. Efficient Cryptographic Schemes Provably as Secure as Subset Sum. J.Crypt., 9(4):199-216, 1996.

12. D. Knuth. The Art of Computer Programming (vol.3): Sorting and Searching. Addison-Wesley, 1973.

13. C.H. Lim and P.J. Lee. More Flexible Exponentiation with Precomputation. CRYPTO'94, LNCS 839, pp.95-107.

14. D. Long and A. Wigderson. The Discrete Log Hides $O(\log n)$ Bits. SIAM J.Computing, 17:363-372, 1988.

15. M. Naor and O. Reingold. Number-Theoretic Constructions of Efficient PseudoRandom Functions. IEEE FOCS, pp.458-467, 1997.

16. S. Patel and G. Sundaram. An Efficient Discrete Log Pseudo Random Generator. CRYPTO'98, LNCS 1462, pp.304-317, 1998.

17. R. Peralta. Simultaneous Security of Bits in the Discrete Log. EUROCRYPT'85, LNCS 219, pp.62-72, 1986.

18. J. Pollard. Monte-Carlo Methods for Index Computation (mod p). Mathematics of Computation, 32(143):918-924, 1978.

19. C. Schnorr Security of Allmost ALL Discrete Log Bits. Electronic Colloquium on Computational Complexity. Report TR98-033. Available at http://www.eccc.uni-trier.de/eccc/.

20. A. Yao. Theory and Applications of Trapdoor Functions. IEEE FOCS, 1982. 